\title{
Rapid Identification of Mycoplasma bovis Strains from Bovine Bronchoalveolar Lavage Fluid with Matrix-Assisted Laser Desorption Ionization-Time of Flight Mass Spectrometry after Enrichment Procedure
}

\author{
Jade Bokma, ${ }^{a}$ Laura Van Driessche, ${ }^{a}$ Piet Deprez, ${ }^{a}$ Freddy Haesebrouck, ${ }^{\mathrm{b}}$ Marianne Vahl,c Eefke Weesendorp, ${ }^{\mathrm{c}}$ \\ Ruud H. Deurenberg, ${ }^{c}$ Bart Pardon, ${ }^{a}$ Filip Boyen ${ }^{b}$

\begin{abstract}
aDepartment of Large Animal Internal Medicine, Faculty of Veterinary Medicine, Ghent University, Merelbeke, Belgium
bDepartment of Pathology, Bacteriology, and Avian Diseases, Faculty of Veterinary Medicine, Ghent University, Merelbeke, Belgium

'Department of Diagnostics and Crisis Organization, Wageningen Bioveterinary Research (WBVR), Lelystad, The Netherlands
\end{abstract}

Filip Boyen and Bart Pardon contributed equally to this work. They jointly supervised this work; however, as most of the experiments were performed in the laboratory of bacteriology, Filip Boyen (bacteriologist) was put as the last author.

ABSTRACT Mycoplasma bovis is a leading cause of pneumonia in modern calf rearing. Fast identification is essential to ensure appropriate antimicrobial therapy. Therefore, the objective of this study was to develop a protocol to identify M. bovis from bronchoalveolar lavage fluid (BALf) with matrix-assisted laser desorption ionization-time of flight mass spectrometry MALDI-TOF MS and to determine the diagnostic accuracy in comparison with other techniques. BALf was obtained from 104 cattle, and the presence of $M$. bovis was determined in the following three ways: (i) rapid identification of $M$. bovis with MALDI-TOF MS (RIMM) (BALf was enriched and after 24, 48, and $72 \mathrm{~h}$ of incubation and was analyzed using MALDI-TOF MS), (ii) triplex real-time PCR for M. bovis, Mycoplasma bovirhinis, and Mycoplasma dispar, and (iii) 10-day incubation on selective-indicative agar. The diagnostic accuracy of the three tests was determined with Bayesian latent class modeling (BLCM). After $24 \mathrm{~h}$ of enrichment, $M$. bovis was identified with MALDI-TOF MS in 3 out of 104 BALf samples. After 48 and $72 \mathrm{~h}$ of enrichment, 32/104 and 38/100 samples, respectively, were $M$. bovis positive. Lipase-positive Mycoplasma-like colonies were seen in 28 of 104 samples. Real-time PCR resulted in 28/104 positive and 12/104 doubtful results for $M$. bovis. The BLCM showed a sensitivity (Se) and specificity (Sp) of $86.6 \%$ (95\% credible interval $[\mathrm{Cl}], 69.4 \%$ to $97.6 \%)$ and $86.4 \%(\mathrm{Cl}, 76.1$ to 93.8$)$ for RIMM. For realtime PCR, Se was $94.8 \%(\mathrm{Cl}, 89.9$ to 97.9$)$ and $\mathrm{Sp}$ was $88.9 \%(\mathrm{Cl}, 78.0$ to 97.4$)$. For selective-indicative agar, Se and $\mathrm{Sp}$ were $70.5 \%(\mathrm{Cl}, 52.1$ to 87.1$)$ and $93.9 \%(\mathrm{Cl}, 85.9$ to 98.4), respectively. These results suggest that rapid identification of $M$. bovis with MALDI-TOF MS after an enrichment procedure is a promising test for routine diagnostics in veterinary laboratories.

KEYWORDS Bayesian latent class model, lipase activity, Mycoplasma bovirhinis, Mycoplasma bovis, Mycoplasma dispar

ycoplasma bovis is one of the primary pathogens causing severe pneumonia in cattle and is also associated with arthritis, otitis, mastitis, and reproductive disorders $(1,2)$. Bovine respiratory disease (BRD) is the leading cause of antimicrobial use in calves $(3,4)$, and $M$. bovis is involved in approximately $20 \%$ to $30 \%$ of pneumonia outbreaks in conventional dairy and beef calves, and almost $100 \%$ of veal calf herds have been in contact with this bacterium (5-7). Rapid diagnosis of $M$. bovis is of great importance to rationally use antimicrobials and limit economic losses, since M. bovis is
Citation Bokma J, Van Driessche L, Deprez P, Haesebrouck F, Vahl M, Weesendorp E, Deurenberg RH, Pardon B, Boyen F. 2020. Rapid identification of Mycoplasma bovis strains from bovine bronchoalveolar lavage fluid with matrix-assisted laser desorption ionizationtime of flight mass spectrometry after enrichment procedure. J Clin Microbiol 58:e00004-20. https://doi.org/10.1128/JCM .00004-20.

Editor Brad Fenwick, University of Tennessee at Knoxville

Copyright $\odot 2020$ American Society for Microbiology. All Rights Reserved. Address correspondence to Jade Bokma jade.bokma@ugent.be.

Received 3 January 2020

Returned for modification 25 January 2020 Accepted 23 March 2020

Accepted manuscript posted online 30 March 2020

Published 26 May 2020 
inherently resistant against widely used antimicrobial agents and is difficult to eradicate once it is chronically present $(1,8)$. In contrast to other Mycoplasma species, M. bovis can be cultured quite well, although it easily takes 5 to 10 days before culture results become available. Also, to obtain definite Mycoplasma spp. identification, other techniques, such as biochemical characterization or PCR, are needed $(9,10)$. This is of great importance, as $M$. bovis is generally recognized as a primary pathogen. However, the pathogenic significance of other Mycoplasma species, such as M. arginini, M. bovirhinis, and $M$. dispar, are more controversial, as they are isolated from both healthy and pneumonic lungs (11-14), and incorrect identification could lead to unnecessary antimicrobial use. Selective-indicative agar using lipase activity as an M. bovis-specific feature has been described to distinguish $M$. bovis from other bovine Mycoplasma spp. $(15,16)$. Unfortunately, its diagnostic performance is currently not known. PCR is the preferred method for final identification of Mycoplasma species. We currently are observing a shift toward PCR identification directly on the specimen, such as bronchoalveolar lavage fluid (BALf), in the case of pneumonia. Even though this is more rapid, due to logistic reasons, laboratories usually collect samples to perform in a weekly or twice weekly analysis, whereupon diagnostic results still take several days.

Matrix-assisted laser desorption ionization-time-of-flight mass spectrometry (MALDITOF MS) is widely used as a rapid, low cost, culture-based diagnostic tool for the identification of bacteria, including Mycoplasma spp. (17). Nevertheless, prior isolation of $M$. bovis on specific solid medium is still necessary, and final identification can take up to 10 days $(10,18,19)$. To reduce sample turnaround time, at present, there is great interest in the identification of bacteria by MALDI-TOF MS directly from the sample or after a short enrichment period in liquid broth as is already done for urine, blood, milk, and BALf specimens (20-23). However, such a technique is currently not available for Mycoplasma spp., presumably because of difficulties such as their fastidious growth and overgrowth by other bacteria.

Therefore, the objective of this study was to develop a protocol to identify M. bovis directly from BALf after an enrichment procedure with MALDI-TOF MS. The accuracy of this diagnostic test was compared with real-time PCR and biochemical characterization (lipase activity) on solid medium in a Bayesian latent class model (BLCM).

\section{MATERIALS AND METHODS}

Development of a protocol for fast MALDI-TOF detection of $M$. bovis in BALf. (i) Determination of an enrichment procedure and antimicrobial concentrations. $M$. bovis concentration in BALf usually ranges from $1.8 \times 10^{3}$ to $1.03 \times 10^{8} \mathrm{CFU} / \mathrm{ml}$ (24), whereas a minimum concentration of $1.0 \times 10^{8} \mathrm{CFU} / \mathrm{ml}$ is necessary to obtain interpretable spectra with MALDI-TOF MS starting from mycoplasma grown in broth $(18,19)$. Therefore, an enrichment procedure seemed necessary to identify $M$. bovis directly from BALf. We explored different broths as described earlier (19) and experimented additionally with different antimicrobials, since overgrowth of $M$. bovis by other bacteria, such as (fecal) contaminants (e.g., Enterobacteriaceae), commensals (e.g., Streptococcus, Staphylococcus, and Enterococcus) or pathogenic bacteria (e.g., Pasteurellaceae) in liquid medium is very likely $(13,25)$.

Starting from a fresh culture, three M. bovis strains obtained from clinical field samples (Mb144, K6, and K7; passaged 3 to 5 times) were cultured in modified basic pleuropneumonialike organism (PPLO)broth (255420; BD Difco, Berkshire, United Kingdom) with inactivated horse serum (25\%; Gibco, Ireland), and technical yeast extract (0.7\%; Bacto, Belgium) supplemented with sodium pyruvate $(0.5 \%$; SigmaAldrich, Germany), ampicillin sodium salt $(520 \mu \mathrm{g} / \mathrm{ml}$; Sigma-Aldrich, Germany) (19), and colistin sulfate (VMD, Belgium) at a concentration of $967 \mathrm{IU} / \mathrm{ml}$, similar to what was described previously $(26,27)$. Then, $1 \mathrm{ml}$ of the $M$. bovis culture with either meropenem (USP reference standard, Sigma-Aldrich, Germany) or vancomycin (vancomycin hydrochloride from Streptomyces orientalis; Sigma-Aldrich, Germany) in final concentrations of $32,16,8,4,2,1,0.5$, and $0 \mu \mathrm{g} / \mathrm{ml}$ was prepared in Eppendorf tubes at a final $M$. bovis concentration of 1 to $3 \times 10^{4} \mathrm{CFU} / \mathrm{ml}(28)$. After $48 \mathrm{~h}$ of incubation $\left(37^{\circ} \mathrm{C}, 5 \% \mathrm{CO}_{2}\right)$, protein extraction was performed as described before (18). Antimicrobial concentrations where the MALDI-TOF MS identification score (ID-score) for $M$. bovis was $\geq 1.7$ were considered to not inhibit successful identification $(18,29)$. The highest antimicrobial concentration that did not inhibit successful $M$. bovis identification with MALDI-TOF MS was chosen for the rapid identification protocol.

(ii) Identification and enrichment protocol with MALDI-TOF MS from BALf. The final protocol is presented in the next paragraphs as part of the diagnostic test study and will be referred to as "rapid identification of M. bovis with MALDI-TOF MS" (RIMM).

Diagnostic test study. (i) Study population and sampling method. A prospective diagnostic test accuracy assay was performed. To detect a difference in sensitivity of 0.90 to 0.70 with $80 \%$ power, a minimum of 103 samples were needed (30). Therefore, a convenience sample of 104 BALf specimens was 
collected for diagnostic purposes as described before (31). Samples were taken from 3-week-old to 4-year-old cows originating from 10 different farms (5 beef [A to $D$ and $F$ ], 3 dairy [E, G, and $H$ ], and 2 veal [I and J]) in Flanders, Belgium, from January 2019 to May 2019. Subsequently, samples were stored at $4^{\circ} \mathrm{C}$ for 3 to 20 hours before culture-based methods (index tests: RIMM and selective-indicative agar) were performed. All samples were stored $\left(-20^{\circ} \mathrm{C}\right)$ before the reference test (real-time PCR) was performed blindly. All procedures were approved by the local ethical committee under approval number EC2019-1.

(ii) Rapid identification of $M$. bovis with MALDI-TOF MS (RIMM). BALf was vigorously vortexed, and $4 \mathrm{ml}$ was inoculated in $8 \mathrm{ml}$ modified basic PPLO broth as described above, supplemented with $32 \mu \mathrm{g} / \mathrm{ml}$ vancomycin and $32 \mu \mathrm{g} / \mathrm{ml}$ meropenem. After 24,48 , and $72 \mathrm{~h}$ of incubation $\left(37^{\circ} \mathrm{C}, 5 \% \mathrm{CO}_{2}\right)$, protein extraction was performed on $1 \mathrm{ml}$ of the enriched BALf culture as described before, and $1 \mu \mathrm{l}$ of lysate was spotted in triplicate on target polished steel barcode (BC) plates $(18,19)$. Spotted samples were air dried and covered with $1 \mu \mathrm{l}$ alpha-cyano-4-hydroxycinnamic acid matrix (Bruker Daltonics, Bremen, Germany). All samples were processed with an Autoflex III Smartbeam MALDI-TOF MS instrument using FlexControl and MBT Compass software (Bruker Daltonics, Bremen, Germany). External calibration and validation were performed by adding bacterial test standard as described by the manufacturer (Bruker Daltonics, Bremen, Germany). Negative controls were performed by adding $1 \mu l$ of the matrix only. The standard library (MBT Compass server version 4.1.90 PYTH) was extended with four in-house main spectrum profiles (MSPs) for M. bovis as outlined before (19) and extra MSPs of M. bovirhinis (NCTC 10118), Mycoplasma ovipneumoniae (NCTC 10151), and M. dispar (NCTC 10125). Identification of Mycoplasma spp. was considered reliable at the species level when logarithmic score values were $\geq 1.7$ as proposed in previous studies $(18,29)$, whereas identification of nonmycoplasmal bacteria was considered reliable at the species level when score values were $\geq 2.0$ and at the genus level when score values were $\geq 1.7$ and $<2.0$ (20).

(iii) Selective-indicative agar. One hundred $\mu$ of 10 -fold dilutions of BALf was inoculated on an in-house modified PPLO agar containing Difco PPLO agar (product number 241210) enriched with 25\% inactivated horse serum (Gibco), 0.7\% technical yeast extract (Bacto), 0.5\% D-(+)-glucose monohydrate (Sigma-Aldrich), $520 \mu \mathrm{g} / \mathrm{ml}$ ampicillin sodium salt (Sigma-Aldrich), $967 \mathrm{lU} / \mathrm{ml}$ colistin sulfate (VMD), and $0.1 \%$ Tween 80 (polysorbate 80; Sigma-Aldrich). Tween 80 was added for observing lipase activity as an indicator for $M$. bovis $(15,16)$. After 1 to 10 days of incubation $\left(37^{\circ} \mathrm{C}, 5 \% \mathrm{CO}_{2}\right)$, presumptive Mycoplasma colonies (fried egg morphology) were identified as $M$. bovis based on the presence of lipase activity, observed as an "oil-like" film surrounding the colonies, and were counted.

(iv) Triplex real-time PCR. A previously described triplex real-time PCR was chosen as the reference test, as this method showed results comparable to other PCR methods used for routine diagnostics to identify M. bovis from $\operatorname{BALf}(32,33)$. The limit of detection was determined at $30 \mathrm{CFU} / \mathrm{ml}$ for $M$. bovis and M. bovirhinis and $300 \mathrm{CFU} / \mathrm{ml}$ for $M$. dispar as described previously (32). BALf samples were thawed before DNA extraction. After vortexing, $200 \mu \mathrm{l}$ of sample was used for DNA extraction with the MagNA Pure 96 Instrument (Roche) using the MagNA Pure 96 DNA and viral NA small volume kit (Roche) for DNA extraction. Then, $5 \mu$ l extracted DNA was used for the triplex real-time PCR detecting M. bovis, M. dispar, and $M$. bovirhinis as described before (32). Fresh $M$. bovis, $M$. dispar, and $M$. bovirhinis cultures from in-house reference strains were used as an internal control to monitor DNA extraction, as well as inhibition of the PCRs. Threshold cycle $\left(C_{T}\right)$ values were interpreted as positive $(<35)$, doubtful $(35$ to 40$)$, or negative $(>40)$ as previously described (33).

(v) Conventional bacterial culture for nonmycoplasmal bacteria. An essential part of the protocol was to avoid overgrowth of $M$. bovis by other pathogens. Therefore, to quantify other fast-growing nonmycoplasmal pathogens and the contamination present in the BALf samples, $100 \mu$ of 10 -fold dilutions of BALf was cultured on Columbia agar supplemented with $5 \%$ sheep blood (blood agar; Oxoid, UK). Nonmycoplasmal bacteria were identified with MALDI-TOF MS after 1 day of incubation $\left(37^{\circ} \mathrm{C}, 5 \%\right.$ $\mathrm{CO}_{2}$ ). Additionally, after $72 \mathrm{~h}$ of enrichment in the BALf in modified PPLO medium, $50 \mu \mathrm{l}$ was cultured on blood agar for $24 \mathrm{~h}\left(37^{\circ} \mathrm{C}, 5 \% \mathrm{CO} 2\right)$ to check for the residual presence of nonmycoplasmal species.

Statistical analysis. (i) Crosstabulation. First, the diagnostic accuracy (sensitivity and specificity) of both the RIMM and the selective-indicative agar (index tests) was determined with real-time PCR as the reference test (WinEpi, Zaragoza, Spain). BALf was considered positive for $M$. bovis when the ID-score of MALDI-TOF MS was $\geq 1.7$ (direct identification) after $72 \mathrm{~h}$ of incubation, when the $C_{T}$ score was $<40$ (real-time PCR), or when Mycoplasma-like colonies showed lipase activity (bacterial culture). For 4 BALf samples, no results for $72 \mathrm{~h}$ of incubation were obtained (due to a practical problem), and for these samples, results from $48 \mathrm{~h}$ were used to compare with real-time PCR and the selective-indicative agar.

(ii) Bayesian latent class modeling. (a) Definition of outcome tested. The sensitivity and specificity of the real-time PCR are not $100 \%(32,33)$. Therefore, and also because of issues with clinical interpretation (detection of a small amount of nonviable pathogens), this test cannot truly be considered a gold standard. To account for this issue, a second statistical analysis was performed to determine the diagnostic accuracy of the three methods. Bayesian latent class models create their own probabilistic definition of the outcome studied, depending on what the tests actually detect. In this study, PCR detects DNA from either living or dead bacteria. The selective-indicative agar detects culturable bacteria with lipase activity. Culture enrichment combined with MALDI-TOF MS detects protein spectra from culturable bacteria. Therefore, in our judgement, the three tests detect three distinguishing parameters, and a model for three independent tests was built. In addition, the model for dependent tests (with both culture-based methods being dependent of each other) was built to compare independent and dependent outcomes.

(b) Model development. In order to assess the accuracy of the three tests-(i) real-time PCR (detection of DNA), (ii) RIMM (detection of proteins), and (iii) selective-indicative agar (detection of lipase activi- 
ty) - to detect the presence of $M$. bovis, a latent class model ( 1 population, 3 tests) was considered, with each test regarded as independent from the others. The unknown parameters of interest were sensitivity and specificity of the three diagnostic tests and the prevalence of $M$. bovis in the study population. Once the likelihood of the process generating the data observation is described, which in this case is a multinomial probability distribution, the estimation of posterior densities can be obtained using the Bayes theorem, which links the likelihood with the posterior distribution (inference). At this stage, prior information on any parameter in the likelihood can be added to obtain posterior densities of the different parameters using a Markov chain Monte-Carlo algorithm (Gibbs sampling). The prior information is a way to narrow parameter uncertainty when previous scientific information is available. In terms of prevalence and the $\mathrm{Se} / \mathrm{Sp}$ of tests, the priors are modeled using beta distributions that are naturally bound from 0 to 1. Priors can be uninformative (any value has same probability of happening) or informative (some values are more or less probable) (34).

(c) Prior distribution determination process. Prior information was derived from the available literature and expert opinion. For both RIMM and the selective-indicative agar, no literature information was available. Previous work on M. bovis real-time PCR estimated an Se of $95.2 \%$ (95\% confidence interval [Cl95], 76.1\% to $99.9 \%)$ and Sp of $73.9 \%$ (Cl95, 51.6\% to $89.8 \%)$ (32). Also, for the prevalence of $M$. bovis in the population, prior information was available $(7,35)$. This literature information was combined with the best guesses of experts in the field (first author and senior authors). The 5 th percentiles were guessed at $90 \%, 95 \%$, and $50 \%$ for Se, Sp, and prevalence, respectively. These values were used to determine the beta distribution parameters of the corresponding prior distribution using a free online beta distribution calculator (Epitools, Sergeant, ESG, Ausvet Animal Health Services and Australian Biosecurity calculator; available at https://epitools.ausvet.com.au/), resulting in beta $(99.7,6.19)$, beta (1), and beta $(6.28,13.32)$ for Se, Sp, and prevalence, respectively.

In total, the three following models were run: the first model with all prior information on all parameters set at uninformative (beta 1,1), the second model with informative priors on the Se and Sp of real-time PCR, and the third model with informative priors on the $M$. bovis prevalence and real-time PCR.

The parameters of interest were determined based on a sample from the posterior distribution using Gibbs sampling with WinBUGS statistical freeware version 1.4.3. (MRC Biostastics Unit, Cambridge, UK). Estimation of posterior densities and model assessment was done using recommended techniques (36). A total of 100,000 iterations were used for each model after a burn-in of 5,000 iterations. Three chains were run with different initial values. The posterior median and 2.5 to 97.5 credible intervals $(\mathrm{Cl})$ were extracted for each parameter. Model convergence was checked by visual inspection of density and Gelman-Rubin plots. Plots of chain autocorrelation were inspected to investigate the need for thinning of chains.

For smaller data sets, informative prior elicitation can be a process that could potentially have an impact on posterior density. Therefore, a sensitivity analysis was performed, running alternative models with very different prior specifications to the main model. It was determined whether posterior estimates of these alternative models were included in the $95 \% \mathrm{Cl}$ of the main model (34).

\section{RESULTS}

Development of a protocol for fast MALDI-TOF detection of $M$. bovis in BALf. Determination of an enrichment procedure and antimicrobial concentrations. After $48 \mathrm{~h}$ of incubation, $M$. bovis strains were identified with MALDI-TOF MS as M. bovis (ID-score, $\geq 2.0$ ) after protein extraction for all tested antimicrobial concentrations. Therefore, a concentration of $32 \mu \mathrm{g} / \mathrm{ml}$ for both meropenem and vancomycin was selected for further testing.

Diagnostic test study. Triplex real-time PCR resulted in 26.9\% (28/104) positive BALf samples $\left(C_{T}, \leq 35\right)$, $11.5 \%(12 / 104)$ were doubtful $\left(35<C_{T}<40\right)$, and $61.5 \%$ (64/104) were negative $\left(C_{T}, \geq 40\right.$ or no detection) for $M$. bovis. For $M$. bovirhinis, $79.8 \%$ (83/104) were positive, $11.5 \%$ (12/104) were doubtful, and $8.7 \%(9 / 104)$ were negative, and for M. dispar, these figures were $92.3 \%$ (96/104), 3.8\% (4/104), and 3.8\% (4/104), respectively (see Table $\mathrm{S} 1$ in the supplemental material).

After $24 \mathrm{~h}$ of enrichment, with the RIMM method, 2.9\% (3/104) of the BALf samples were positive for M. bovis, and after 48 and 72 h, 30.7\% (32/104) and 38.0\% (38/100), respectively, were positive. For 4 samples, no results were obtained after $72 \mathrm{~h}$ of incubation, because of a practical problem. ID-scores for $M$. bovis ranged from 1.74 to 2.65 and are shown in Table S1. M. bovirhinis was identified after $24 \mathrm{~h}$ (BALf sample no. 18, 23, 28, 60, and 95), 48 h (BALf sample no. 26, 28, 29, 32, 35, 38, 43, 60, 64, and 102), and $72 \mathrm{~h}$ (BALf sample no. 60,63, 66, and 88) with ID-scores of 1.70 to 1.88 . In BALf sample no. 24, M. ovipneumoniae was identified after 48 and $72 \mathrm{~h}$ of incubation (ID-score, 1.78 and 1.72, respectively). M. dispar was not detected with the RIMM method in any sample throughout the experiment. 
TABLE $12 \times 2$ contingency table for direct MALDI-TOF MS identification as index test compared to real-time PCR as reference test for identification of Mycoplasma bovis from BALf samples $(n=104)$

\begin{tabular}{|c|c|c|c|c|}
\hline \multirow[b]{2}{*}{ Test } & \multirow{2}{*}{$\begin{array}{l}\text { Result } \\
\text { category }\end{array}$} & \multicolumn{2}{|c|}{$\begin{array}{l}\text { Reference test results } \\
\text { (real-time PCR) (\%) (no.) }\end{array}$} & \multirow{2}{*}{$\begin{array}{l}\text { Total no. } \\
\text { of tests }\end{array}$} \\
\hline & & Positive & Negative & \\
\hline \multirow[t]{3}{*}{ MALDI-TOF MS } & Positive & $75(30 / 40)$ & $14(9 / 64)$ & 39 \\
\hline & Negative & $25(10 / 40)$ & $86(55 / 64)$ & 65 \\
\hline & Total no. & 40 & 64 & 104 \\
\hline \multirow[t]{3}{*}{ Selective-indicative agar } & Positive & $60(24 / 40)$ & $6(4 / 64)$ & 28 \\
\hline & Negative & $40(16 / 40)$ & $94(60 / 64)$ & 76 \\
\hline & Total no. & 40 & 64 & 104 \\
\hline
\end{tabular}

Out of 104 samples, 28 (26.9\%) showed lipase-positive Mycoplasma-like colonies on the selective-indicative agar (Table S1), ranging from $1.0 \times 10^{1}$ to $4.6 \times 10^{4} \mathrm{CFU} / \mathrm{ml}$ BALf (mean, $3.8 \times 10^{3}$; median, $3.3 \times 10^{2}$ ). Of those 104 BALf samples, $18 \%$ (19/104) did not show additional bacterial growth on blood agar plates, whereas $81.7 \%$ (85/104) did, among which were multiple pathogens and commensals (Table S1). Despite the presence of these nonmycoplasmal bacteria in the original BALf samples, the selective enrichment phase did not allow nonmycoplasmal bacterial growth to an extent that was detected by MALDI-TOF MS.

Residual contamination was checked after $72 \mathrm{~h}$ of enrichment. There was no microbial growth on blood agar in $70 \%$ of the BALf samples after $24 \mathrm{~h}$ of incubation (70/100). For the samples with microbial growth on blood agar, MALDI-TOF MS identified Candida spp. (4/30), Aspergillus fumigatus (2/30), Stenotrophomonas maltophilia (1/30), and Staphylococcus spp. (1/30). In 23 out of 30 blood agars showing microbial growth, no identification was possible with MALDI-TOF MS, but their macroscopic appearance suggested mainly fungal contaminants.

The $2 \times 2$ contingency tables of the RIMM method and the selective-indicative agar compared with real-time PCR as the reference test are shown in Table 1. The sensitivity (Se) and specificity (Sp) for the RIMM compared to real-time PCR were $75.0 \%$ (CI95, $61.6 \%$ to $88.4 \%)$ and $85.9 \%$ (C195, $77.4 \%$ to $94.5 \%)$, respectively. The selective-indicative agar showed a $60.0 \%$ (Cl95, $44.8 \%$ to $75.2 \%)$ Se and a $93.8 \%$ (Cl95, $87.8 \%$ to $99.7 \%)$ Sp compared to the real-time PCR test.

All latent class models converged, and the prior distributions and posterior summary statistics of each model are shown in Table 2. Parameters were particularly stable between models, and the results obtained from the sensitivity analysis were highly robust to changes in the prior distributions. Model 3 included the most prior informa-

TABLE 2 Posterior median and 95\% Cl of three independent Bayesian latent class models for the prevalence of $M$. bovis, sensitivity and specificity of the RIMM, triplex real-time PCR and selective-indicative agar used to diagnose M. bovis from bronchoalveolar lavage fluid samples

\begin{tabular}{|c|c|c|c|c|c|c|}
\hline \multirow[b]{2}{*}{ Parameter ${ }^{a}$} & \multicolumn{2}{|l|}{ Model $1^{b}$} & \multicolumn{2}{|l|}{ Model $2^{c}$} & \multicolumn{2}{|l|}{ Model $3^{d}$} \\
\hline & Prior densities & $\begin{array}{l}\text { Posterior densities } \\
(95 \% \mathrm{Cl})\end{array}$ & Prior densities & $\begin{array}{l}\text { Posterior densities } \\
(95 \% \mathrm{Cl})\end{array}$ & Prior densities & $\begin{array}{l}\text { Posterior densities } \\
(95 \% \mathrm{Cl})\end{array}$ \\
\hline$S p_{p c r}$ & Beta (1) & $89.2(78.1-97.8)$ & Beta (1) & 89.1 (78.1-97.7) & Beta $(1)$ & 88.9 (78.0-97.4) \\
\hline $\mathrm{Se}_{\text {rimm }}$ & Beta (1) & $86.0(68.3-97.5)$ & Beta (1) & $86.3(68.8-97.5)$ & Beta (1) & $86.6(69.4-97.6)$ \\
\hline $\mathrm{Sp}_{\text {rimm }}$ & Beta (1) & $86.8(76.3-94.9)$ & Beta (1) & 86.5 (76.2-93.9) & Beta (1) & $86.4(76.1-93.8)$ \\
\hline $\mathrm{Se}_{\text {sia }}$ & Beta (1) & $69.5(50.8-86.7)$ & Beta (1) & $70.2(51.8-86.9)$ & Beta (1) & $70.5(52.1-87.1)$ \\
\hline
\end{tabular}

${ }^{a}$ pcr, triplex real-time PCR; Prev, prevalence of M. bovis; rimm, rapid identification of M. bovis with MALDI-TOF MS method; Se, sensitivity; sia, selective-indicative agar; Sp, specificity.

${ }^{b}$ Model 1, no informative priors.

cModel 2, informative priors on prevalence and $\mathrm{Se}_{\mathrm{pcr}}$ (mode, 95\%; 5th percentile, 90\%) and $\mathrm{Sp}_{\mathrm{pcr}}$ (mode, 74\%; 5th percentile, 95\%) (30).

${ }^{d}$ Model 3, informative prior on $\mathrm{Se}_{\mathrm{pcr}}, \mathrm{Sp}_{\mathrm{pcr}}$, and prevalence of M. bovis in BALf (mode. 30\%; 5th percentile; 50\%) (33). 
tion and is therefore expected to be the most accurate. The prevalence of $M$. bovis was $32.6 \%(\mathrm{Cl}, 23.5 \%$ to $42.6 \%)$, which was comparable to the prior information added to the third model. In independent model 3, RIMM showed an Se and Sp of $86.6 \%(\mathrm{Cl}$, $69.4 \%$ to $97.6 \%)$ and $86.4 \%(\mathrm{Cl}, 76.1 \%$ to $93.8 \%)$, respectively. Real-time PCR had an Se of $94.8 \%(\mathrm{Cl}, 89.9 \%$ to $97.9 \%)$ and an $\mathrm{Sp}$ of $88.9 \%(\mathrm{Cl}, 78.0 \%$ to $97.4 \%)$. The selectiveindicative agar had an Se and Sp of $70.5 \%(\mathrm{Cl}, 52.1 \%$ to $87.1 \%)$ and $93.9 \%(\mathrm{Cl}, 85.9 \%$ to $98.4 \%)$, respectively. No significant differences between the independent and dependent models were observed.

\section{DISCUSSION}

The objective of the present study was to achieve a proof of concept for rapid identification of $M$. bovis from bovine BALf samples after enrichment with MALDI-TOF MS and to compare these with another culture-based method (selective-indicative agar) and a DNA-based reference test (real-time PCR). In this study, we identified $M$. bovis from the majority of BALf samples with RIMM within 2 days after incubation, and even more after 3 days. The current protocol reduced the relevant growth of nonmycoplasmal bacteria and nonpathogenic Mycoplasma spp. present in BALf, resulting in high sensitivity (86.6\%; $\mathrm{Cl}, 69.4$ to 97.6$)$ and specificity $(86.4 \% ; \mathrm{Cl}, 76.1$ to 93.8 ) of this diagnostic test as determined by BLCM. Prior information of the BLMC was extracted from peer-reviewed journals, and the sensitivity analysis for robustness of all models was verified. These methods together make the possibility of bias due to the best guesses of the experts in the field less likely.

False negatives of the RIMM method compared to real-time PCR can be explained by the viability of $M$. bovis. PCR detects DNA, while for culture-based methods, such as MALDI-TOF MS, bacteria need to be alive. Some of the sampled calves might have been treated with antimicrobials before BALf samples were obtained, resulting in nonviable $M$. bovis in the lungs, so only DNA could be detected. This would suggest that no active $M$. bovis infection is currently present, and the clinical relevance of positive results from the real-time PCR might be questioned, in contrast to culture-based methods (RIMM and selective-indicative agar). In addition, cross-reaction with Mycoplasma agalactiae has been described for the currently used real-time PCR (32), while MALDI-TOF MS has been described to accurately distinguish $M$. bovis and $M$. agalactiae $(17,18)$. Both previous arguments therefore more likely result in a false-positive PCR result rather than a false-negative culture-based result, and therefore the current specificity of the RIMM might be underestimated.

Disagreement between a negative result for $M$. bovis with real-time PCR but a positive result with the RIMM method might be explained by the fact that the BALf volume used for RIMM was $4 \mathrm{ml}$, while the volume was only $200 \mu \mathrm{l}$ for the real-time PCR reference test and $100 \mu \mathrm{l}$ for biochemical identification (lipase activity) on agar. BALf contains mucus clumps and cells, which could lead to a heterogenic suspension of $M$. bovis. Although samples were vortexed vigorously, it is possible that certain parts of the BALf did not end up in the aliquot for real-time PCR. This could have caused a higher chance of isolation using the RIMM method compared with detection using real-time PCR or selective-indicative agar. The ability to process larger volumes with the RIMM method from BALf without extra labor is an advantage over both other tests (real-time PCR and selective-indicative agar) and might even increase the sensitivity of culturebased methods. Considering (i) that most clinical samples in this study contained $10^{2}$ to $10^{3} \mathrm{CFU} / \mathrm{ml}$, (ii) the generation time of $M$. bovis (2 $\mathrm{h}$ in exponential phase) (37), and (iii) that the detection limit of the MALDI-TOF MS is $10^{8} \mathrm{CFU} / \mathrm{ml}$ (18), detection of $M$. bovis from BALf after $48 \mathrm{~h}$ of incubation can be expected and is in line with the observations. For samples in which $M$. bovis was detected after $72 \mathrm{~h}$ of incubation at the earliest, for example, the presence of other pathogens, mucus composition, number of inflammatory cells, or other antimicrobial substances might have influenced the $M$. bovis growth rate or MALDI-TOF MS identification efficacy.

Various antimicrobials were added to the modified PPLO broth, as high antimicrobial resistance levels against different antimicrobials were observed in bacteria isolated 
from cattle (38-40). Meropenem was considered due to its broad spectrum and strong activity against Gram-negative bacteria, although this would probably not suppress all bacterial growth in BALf, as for example, methicillin-resistant Staphylococcus aureus (MRSA) shows resistance against meropenem in humans but is also a common pathogen in calves (41). Therefore, vancomycin was used as well. Until now, only very low levels to no resistance is detected against this antibiotic in MRSA and Enterococcus strains obtained from cattle $(42,43)$. It should be kept in mind that critically important antibiotics, even though used under laboratory circumstances, should be properly disposed of after use.

Even though the current method was able to suppress nonmycoplasmal bacterial growth, there is still room for improvement for the reduction of yeast and fungal growth. In five of the BALf samples with a false-negative MALDI-TOF MS result compared to real-time PCR, fungal growth was observed, and this might have caused interference with identification of $M$. bovis, as fungal pigments can suppress the desorption process (44) or may lead to the presence of interfering peaks. Adding an antimycotic drug, such as amphotericin B, might help increase the sensitivity of the rapid MALDI-TOF MS identification method (45).

The real-time PCR cannot be seen as the gold standard technique for several reasons. First, sensitivity and specificity are not 100\% (32). Second, studies concluded that the sensitivity of culture was sometimes higher than that of real-time PCR assays $(24,33)$, and in this study $11.5 \%$ of the real-time PCR results were doubtful and therefore difficult to compare to the culture-based methods where culture was either positive or negative. Therefore, a third test was included in this study to perform a BLCM, as is recommended when no gold standard is available (36).

The selective-indicative agar using lipase activity to identify $M$. bovis is not widely used and is therefore somewhat controversial. Though national laboratories in Belgium have used this method for years, this study is the first to show its diagnostic accuracy. This method resulted in a moderate sensitivity (70.5\%) on the one hand, but on the other hand, a specificity (93.8\%) that was even higher than real-time PCR (88.9\%). It has been described that not all $M$. bovis strains show lipase activity, which could explain false negatives, whereas some other Mycoplasma spp. do possess this characteristic, which could result in false positives (46). Nevertheless, current results show that this medium might be helpful in M. bovis screenings where low cost is necessary and less experienced staff is located. It could also be helpful in choosing relevant colonies on agar plates for subsequent identification methods, such as real-time PCR. In addition, it cannot be ruled out that the use of other selective-indicative agar media, commercially available or not, could have resulted in different sensitivity or specificity data.

Other Mycoplasma spp. were identified as well in the BALf. Real-time PCR showed that at least $79.8 \%$ of the BALf samples were positive for M. bovirhinis, and $92.3 \%$ were positive for $M$. dispar. However, only $15.4 \%$ were positive for $M$. bovirhinis using the RIMM method with MALDI-TOF MS after $72 \mathrm{~h}$ of enrichment. Mixtures of $M$. bovis, $M$. bovirhinis, and $M$. dispar in BALf of cattle are common (11). Real-time PCR might have overestimated the prevalence of $M$. bovirhinis and $M$. dispar, because $M$. bovirhinis PCR cross-reacts with $M$. canis, and M. dispar PCR cross-reacts with Acholeplasma axanthum and Mycoplasma alkalescens (32). However, cross-reaction can probably not explain the large difference observed between the RIMM method and the real-time PCR. The enrichment medium used in this study seemed to preselect for $M$. bovis growth. $M$. bovirhinis and $M$. dispar are glucose fermenting, while in our medium only pyruvate was added as a carbon source (47). The latter is a great advantage in the diagnosis of $M$. bovis, as $M$. bovirhinis and $M$. bovis can both be identified with MALDI-TOF MS after 2 days of incubation (17). We did, however, observe a shift in identification of $M$. bovirhinis, where two samples were positive after $24 \mathrm{~h}$ and negative after $48 \mathrm{~h}$. As the medium was not optimal for $M$. bovirhinis, one reason for this disparity could be that the concentration of (viable) $M$. bovirhinis balanced around the detection limit of the MALDI-TOF MS. Another reason might be that after $24 \mathrm{~h}$, the concentration of $M$. bovis became higher than the concentration of M. bovirhinis, as Pereyre et al. (18) confirmed 
that $M$. bovis was the only pathogen recognized by MALDI-TOF MS when samples contained 2 to 3 Mycoplasma species. Irrelevant $M$. dispar growth is less of a concern in culture-based methods, as this is a fastidious grower and is more difficult to isolate (47). Identification of $M$. ovipneumoniae from bovine BALf was unexpected. However, a recent study showed this species to be abundantly present in bovine BALf as well (48). The clinical relevance in cattle is unknown, although $M$. ovipneumoniae infections can have serious consequences in small ruminants, such as pneumonia, decreases in lamb production, and decreases in average daily gain $(49,50)$. Even though the MALDI-TOF MS specificity for accurate $M$. ovipneumoniae detection is not described, Spergser et al. (17) tested 19 M. ovipneumoniae clinical isolates against their own in-house library, which resulted in $100 \%$ identification with a score value of $\geq 1.7$ (17).

MALDI-TOF MS is already proven to be of assistance for the identification of human and veterinary mycoplasmas from culture $(17,18,29)$. In the future, there might be a great opportunity to develop a rapid and specific diagnostic tool to identify other pathogenic Mycoplasma spp. from BALf as well (for example, M. ovipneumoniae from small ruminants or Mycoplasma pneumoniae from humans) and accelerate turnover time in pneumonia diagnostics.

The current study shows that identification of $M$. bovis from BALf with the RIMM method is possible within 48 to $72 \mathrm{~h}$ after sampling. Compared to real-time PCR, RIMM is probably less expensive, the clinical relevance might be higher, and when desired, it holds the opportunity to perform additional susceptibility testing and strain typing (51, 52). Therefore, rapid identification of $M$. bovis with MALDI-TOF MS is a promising method for diagnosis of $M$. bovis in veterinary laboratories. However, it is necessary for laboratories using this approach for the detection of $M$. bovis from clinical samples to fully validate or comprehensively qualify this method. The validation parameters should include accuracy, precision, linearity and range of measurement, specificity, limit of detection, limit of quantitation, and robustness.

\section{SUPPLEMENTAL MATERIAL}

Supplemental material is available online only.

SUPPLEMENTAL FILE 1, PDF file, 0.2 MB.

\section{ACKNOWLEDGMENTS}

We thank the members of the Department of Diagnostics and Crisis Organization, Wageningen Bioveterinary Research, for helping with the real-time PCR. Additionally, we thank the National Infection Service Department of Public Health England (London) for providing the MSPs for several Mycoplasma species. Also, Arlette Van de Kerckhove and Serge Verbanck are greatly appreciated for preparing the modified PPLO agar and broths.

J.B. is supported by a grant from the Belgian Federal Public Service, Health, Food Chain Safety and Environment (RF 17/6313, MALDIRESP/MA). The MALDI-TOF MS was financed by the Research Foundation Flanders (FWO-Vlaanderen) as a Hercules project $(\mathrm{GOH} 2516 \mathrm{~N}, \mathrm{AUGE} / 15 / 05)$. B.P. has received honoraria for acting as a speaker or consultant for pharmaceutical (Zoetis, MSD, Vetoquinol, Dopharma, Boehringer Ingelheim, Dechra, Hipra, Ceva, Merial, and Elanco) and agricultural (Boerenbond and Algoet nutrition) companies. The funders had no role in study design, data collection and interpretation, or the decision to submit the work for publication.

\section{REFERENCES}

1. Maunsell FP, Donovan GA. 2009. Mycoplasma bovis infections in young calves. Vet Clin North Am Food Anim Pract 25:139-177. https://doi.org/ 10.1016/j.cvfa.2008.10.011.

2. Maunsell FP, Woolums AR, Francoz D, Rosenbusch RF, Step DL, Wilson DJ, Janzen ED. 2011. Mycoplasma bovis infections in cattle. J Vet Intern Med 25:772-783. https://doi.org/10.1111/j.1939-1676.2011 .0750.x.

3. Pardon B, Catry B, Dewulf J, Persoons D, Hostens M, De Bleecker K,
Deprez P. 2012. Prospective study on quantitative and qualitative antimicrobial and anti-inflammatory drug use in white veal calves. J Antimicrob Chemother 67:1027-1038. https://doi.org/10.1093/jac/dkr570.

4. Lava M, Schüpbach-Regula G, Steiner A, Meylan M. 2016. Antimicrobial drug use and risk factors associated with treatment incidence and mortality in Swiss veal calves reared under improved welfare conditions. Prev Vet Med 126:121-130. https://doi.org/10.1016/j.prevetmed.2016.02 .002 . 
5. Pardon B, De Bleecker K, Dewulf J, Callens J, Boyen F, Catry B, Deprez P. 2011. Prevalence of respiratory pathogens in diseased, non-vaccinated, routinely medicated veal calves. Vet Rec 169:278-278. https://doi.org/ 10.1136/vr.d4406.

6. Francoz D, Buczinski S, Bélanger AM, Forté G, Labrecque O, Tremblay D, Wellemans V, Dubuc J. 2015. Respiratory pathogens in Québec dairy calves and their relationship with clinical status, lung consolidation, and average daily gain. J Vet Intern Med 29:381-387. https://doi.org/10 $.1111 /$ jvim. 12531 .

7. Pardon B, Callens J, Maris J, Allais L, Van Praet W, Deprez P, Ribbens S. 2020. Pathogen specific risk factors in acute outbreaks of respiratory disease in calves. J Dairy Sci 130:2556-2566.

8. Gautier-Bouchardon AV. 2018. Antimicrobial resistance in Mycoplasma spp. Microbiol Spectr 6:ARBA-0030-2018.

9. Calcutt MJ, Lysnyansky I, Sachse K, Fox LK, Nicholas RAJ, Ayling RD. 2018. Gap analysis of Mycoplasma bovis disease, diagnosis and control: an aid to identify future development requirements. Transbound Emerg Dis 65(Suppl 1):91-109. https://doi.org/10.1111/tbed.12860.

10. Parker AM, Sheehy PA, Hazelton MS, Bosward KL, House JK. 2018. A review of mycoplasma diagnostics in cattle. J Vet Intern Med 32:1241-1252. https:// doi.org/10.1111/jvim.15135.

11. Thomas A, Ball H, Dizier I, Trolin A, Bell C, Mainil J, Linden A. 2002. Isolation of Mycoplasma species from the lower respiratory tract of healthy cattle and cattle with respiratory disease in Belgium. Vet Rec 151:472-476. https://doi.org/10.1136/vr.151.16.472.

12. Bottinelli M, Passamonti F, Rampacci E, Stefanetti V, Pochiero L, Coletti M, Rueca F, Hyatt DR, Schnee C. 2017. DNA microarray assay and real-time $\mathrm{PCR}$ as useful tools for studying the respiratory tract Mycoplasma populations in young dairy calves. J Med Microbiol 66: 1342-1349. https://doi.org/10.1099/jmm.0.000571.

13. Nicola I, Cerutti F, Grego E, Bertone I, Gianella P, D'Angelo A, Peletto S, Bellino C. 2017. Characterization of the upper and lower respiratory tract microbiota in Piedmontese calves. Microbiome 5:152. https://doi.org/10 .1186/s40168-017-0372-5.

14. Timsit E, Workentine M, van der Meer F, Alexander T. 2018. Distinct bacterial metacommunities inhabit the upper and lower respiratory tracts of healthy feedlot cattle and those diagnosed with bronchopneumonia. Vet Microbiol 221:105-113. https://doi.org/10.1016/j.vetmic.2018 .06.007.

15. Shimizu T. 1983. Selective medium for the isolation of Mycoplasma bovis from nasal discharges of pneumonic calves. Res Vet Sci 34:371-373. https://doi.org/10.1016/S0034-5288(18)32241-0.

16. Devriese LA, Haesebrouck F. 1991. Antibiotic susceptibility testing of Mycoplasma bovis using Tween 80 hydrolysis as an indicator of growth. J Vet Med Ser B 38:781-783. https://doi.org/10.1111/j.1439-0450.1991 .tb00943.x.

17. Spergser J, Hess C, Loncaric I, Ramírez AS. 2019. Matrix-assisted laser desorption ionization-time of flight mass spectrometry is a superior diagnostic tool for the identification and differentiation of mycoplasmas isolated from animals. J Clin Microbiol 57:e00316-19. https://doi.org/10 .1128/JCM.00316-19.

18. Pereyre S, Tardy F, Renaudin H, Cauvin E, Del Pra Netto Machado L, Tricot A, Benoit F, Treilles M, Bebear C. 2013. Identification and subtyping of clinically relevant human and ruminant mycoplasmas by use of matrixassisted laser desorption ionization-time of flight mass spectrometry. J Clin Microbiol 51:3314-3323. https://doi.org/10.1128/JCM.01573-13.

19. Bokma J, Pardon B, Van Driessche L, Gille L, Deprez P, Haesebrouck F, Boyen F. 2019. Optimizing identification of Mycoplasma bovis by MALDITOF MS. Res Vet Sci 125:185-188. https://doi.org/10.1016/j.rvsc.2019.06 .010 .

20. Ferreira L, Sánchez-Juanes F, Muñoz-Bellido JL, González-Buitrago JM. 2011. Rapid method for direct identification of bacteria in urine and blood culture samples by matrix-assisted laser desorption ionization time-of-flight mass spectrometry: intact cell vs. extraction method. Clin Microbiol Infect 17:1007-1012. https://doi.org/10.1111/j.1469-0691.2010 .03339.x.

21. Lallemand E, Arvieux C, Coiffier G, Polard JL, Albert JD, Guggenbuhl P, Jolivet-Gougeon A. 2017. Use of MALDI-TOF mass spectrometry after liquid enrichment (BD BactecTM) for rapid diagnosis of bone and joint infections. Res Microbiol 168:122-129. https://doi.org/10.1016/j.resmic 2016.09.005.

22. Barreiro JR, Gonçalves JL, Grenfell R, Leite RF, Juliano L, Santos MV. 2018. Direct identification of bovine mastitis pathogens by matrix-assisted laser desorption/ionization time-of-flight mass spectrometry in pre- incubated milk. Brazilian J Microbiol 49:801-807. https://doi.org/10 .1016/j.bjm.2018.04.012.

23. Van Driessche L, Bokma J, Deprez P, Haesebrouck F, Boyen F, Pardon B. 2019. Rapid identification of respiratory bacterial pathogens from bronchoalveolar lavage fluid in cattle by MALDI-TOF MS. Sci Rep 9:18381. https://doi.org/10.1038/s41598-019-54599-9.

24. Castillo-Alcala F, Bateman KG, Cai HY, Schott CR, Parker L, Clark ME, McRaild P, McDowall RM, Foster RA, Archambault M, Caswell JL. 2012. Prevalence and genotype of Mycoplasma bovis in beef cattle after arrival at a feedlot. Am J Vet Res 73:1932-1943. https://doi.org/10.2460/ajvr.73 .12 .1932 .

25. Sung JY, Hwang Y, Shin MH, Park MS, Lee SH, Yong D, Lee K. 2018. Utility of conventional culture and MALDI-TOF MS for identification of microbial communities in bronchoalveolar lavage fluid in comparison with the GS Junior next generation sequencing system. Ann Lab Med 38: 110-118. https://doi.org/10.3343/alm.2018.38.2.110.

26. Angulo AF, Jacobs MV, van Damme EH, Akkermans AM, de KruijffKroesen I, Brugman J. 2003. Colistin sulfate as a suitable substitute of thallium acetate in culture media intended for mycoplasma detection and culture. Biologicals 31:161-163. https://doi.org/10.1016/S1045-1056 (03)00031-9.

27. Yassin MH, Al-Humiany AA, Mansour A. 2012. Colistin sulphate as a substitute of thallium acetate in culture media for isolation of Mycoplasma from cattle diseased by Mycoplasmosis. Int J Microbiol 3:128-132.

28. Kanci A, Wawegama NK, Marenda MS, Mansell PD, Browning GF, Markham PF. 2017. Reproduction of respiratory mycoplasmosis in calves by exposure to an aerosolised culture of Mycoplasma bovis. Vet Microbiol 210:167-173. https://doi.org/10.1016/j.vetmic.2017.09.013.

29. Randall LP, Lemma F, Koylass M, Rogers J, Ayling RD, Worth D, Klita M, Steventon A, Line K, Wragg P, Muchowski J, Kostrzewa M, Whatmore AM. 2015. Evaluation of MALDI-TOF as a method for the identification of bacteria in the veterinary diagnostic laboratory. Res Vet Sci 101:42-49. https://doi.org/10.1016/j.rvsc.2015.05.018.

30. Bujang MA, Adnan TH. 2016. Requirements for minimum sample size for sensitivity and specificity analysis. J Clin Diagnostic Res 10:YE01-YE06.

31. Van Driessche L, Valgaeren BR, Gille L, Boyen F, Ducatelle R, Haesebrouck F, Deprez P, Pardon B. 2017. A deep nasopharyngeal swab versus nonendoscopic bronchoalveolar lavage for isolation of bacterial pathogens from preweaned calves with respiratory disease. J Vet Intern Med 31:946-953. https://doi.org/10.1111/jvim.14668.

32. Cornelissen J, de Bree FM, van der Wal FJ, Kooi EA, Koene MGJ, Bossers A, Smid B, Antonis AF, Wisselink HJ. 2017. Mycoplasma detection by triplex real-time $P C R$ in bronchoalveolar lavage fluid from bovine respiratory disease complex cases. BMC Vet Res 13:97. https://doi.org/10 .1186/s12917-017-1023-6.

33. Wisselink HJ, Smid B, Plater J, Ridley A, Andersson AM, Aspán A, Pohjanvirta T, Vähänikkilä N, Larsen H, Høgberg J, Colin A, Tardy F. 2019. A European interlaboratory trial to evaluate the performance of different PCR methods for Mycoplasma bovis diagnosis. BMC Vet Res 15:86. https://doi.org/10.1186/s12917-019-1819-7.

34. Branscum AJ, Gardner IA, Johnson WO. 2005. Estimation of diagnostictest sensitivity and specificity through Bayesian modeling. Prev Vet Med 68:145-163. https://doi.org/10.1016/j.prevetmed.2004.12.005.

35. Gille L, Callens J, Supré K, Boyen F, Haesebrouck F, Van Driessche L, van Leenen K, Deprez P, Pardon B. 2018. Use of a breeding bull and absence of a calving pen as risk factors for the presence of Mycoplasma bovis in dairy herds. J Dairy Sci 101:8284-8290. https://doi.org/10.3168/jds.2018 $-14940$.

36. Kostoulas $P$, Nielsen SS, Branscum AJ, Johnson WO, Dendukuri N, Dhand NK, Toft N, Gardner IA. 2017. STARD-BLCM: standards for the reporting of diagnostic accuracy studies that use Bayesian latent class models. Prev Vet Med 138:37-47. https://doi.org/10.1016/j.prevetmed.2017.01 .006 .

37. Bürgi N, Josi C, Bürki S, Schweizer M, Pilo P. 2018. Mycoplasma bovis co-infection with bovine viral diarrhea virus in bovine macrophages. Vet Res 49:2. https://doi.org/10.1186/s13567-017-0499-1.

38. Graveland H, Wagenaar JA, Heesterbeek H, Mevius D, van Duijkeren E, Heederik D. 2010. Methicillin resistant staphylococcus aureus ST398 in veal calf farming: human MRSA carriage related with animal antimicrobial usage and farm hygiene. PLoS One 5:e10990. https://doi.org/10 .1371/journal.pone.0010990.

39. Catry B, Dewulf J, Maes D, Pardon B, Callens B, Vanrobaeys M, Opsomer G, De Kruif A, Haesebrouck F. 2016. Effect of antimicrobial consumption and production type on antibacterial resistance in the bovine respiratory 
and digestive tract. PLoS One 11:e0146488. https://doi.org/10.1371/ journal.pone.0146488.

40. Hordijk J, Fischer EAJ, Van Werven T, Sietsma S, Van Gompel L, Timmerman AJ, Spaninks MP, Heederik DJJ, Nielen M, Wagenaar JA, Stegeman A. 2019. Dynamics of faecal shedding of ESBL- or AmpC-producing Escherichia coli on dairy farms. J Antimicrob Chemother 74:1531-1538. https://doi.org/10.1093/jac/dkz035.

41. Watanabe A, Tokue $Y$, Takahashi $H$, Kikuchi T, Kobayashi T, Gomi K, Fujimura S, Yasui S, Murayama Y, Nukiwa T. 2001. Comparative in-vitro activity of carbapenem antibiotics against respiratory pathogens isolated between 1999 and 2000. J Infect Chemother 7:267-271. https:// doi.org/10.1007/s101560170026.

42. Barlow RS, McMillan KE, Duffy LL, Fegan N, Jordan D, Mellor GE. 2017. Antimicrobial resistance status of Enterococcus from Australian cattle populations at slaughter. PLoS One 12:e0177728. https://doi.org/10 .1371 /journal.pone.0177728.

43. de Jong A, Simjee S, Rose M, Moyaert H, El Garch F, Youala M, EASSA Study Group. 2019. Antimicrobial resistance monitoring in commensal enterococci from healthy cattle, pigs and chickens across Europe during 2004-14 (EASSA Study). J Antimicrob Chemother 74:921-930. https://doi .org/10.1093/jac/dky537.

44. Buskirk AD, Hettick JM, Chipinda I, Law BF, Siegel PD, Slaven JE, Green BJ, Beezhold DH. 2011. Fungal pigments inhibit the matrix-assisted laser desorption/ionization time-of-flight mass spectrometry analysis of darkly pigmented fungi. Anal Biochem 411:122-128. https://doi.org/10.1016/j .ab.2010.11.025.

45. Arai S, Yoshida K, Izawa A, Kumagai K, Ishida N. 1966. Effect of antibiotics on growth of Mycoplasma pneumonia Mac. J Antibiot 19:118-120.
46. Rottem S, Razin S. 1964. Lipase activity of Mycoplasma. J Gen Microbiol 37:123-134. https://doi.org/10.1099/00221287-37-1-123.

47. Tully JG, Whitcomb RF (ed). 1979. The mycoplasmas, vol II: human and animal mycoplasmas. Academic Press, New York, NY.

48. Klima CL, Holman DB, Ralston BJ, Stanford K, Zaheer R, Alexander TW, McAllister TA. 2019. Lower respiratory tract microbiome and resistome of bovine respiratory disease mortalities. Microb Ecol 78:446-456. https://doi.org/10.1007/s00248-019-01361-3.

49. Lindström L, Tauni FA, Vargmar K. 2018. Bronchopneumonia in Swedish lambs: a study of pathological changes and bacteriological agents. Acta Vet Scand 60:54. https://doi.org/10.1186/s13028-018-0409-1.

50. Manlove K, Branan M, Baker K, Bradway D, Cassirer EF, Marshall KL, Miller RS, Sweeney S, Cross PC, Besser TE. 2019. Risk factors and productivity losses associated with Mycoplasma ovipneumoniae infection in United States domestic sheep operations. Prev Vet Med 168:30-38. https://doi .org/10.1016/j.prevetmed.2019.04.006.

51. Van Driessche L, Bokma J, Gille L, Ceyssens PJ, Sparbier K, Haesebrouck F, Deprez P, Boyen F, Pardon B. 2018. Rapid detection of tetracycline resistance in bovine Pasteurella multocida isolates by MALDI Biotyper antibiotic susceptibility test rapid assay (MBT-ASTRA). Sci Rep 8:13599. https://doi.org/10.1038/s41598-018-31562-8.

52. Hata E, Harada T, Itoh M. 2019. Relationship between antimicrobial susceptibility and multilocus sequence type of Mycoplasma bovis isolates and development of a method for rapid detection of point mutations involved in decreased susceptibility to macrolides, lincosamides, tetracyclines, and spectinomycin. Appl Environ Microbiol 85:e00575-19. https://doi.org/10.1128/AEM.00575-19. 